EOMmun Communication et organisation

Ory

Structure et communication

Les structures de communication

Valérie Carayol

(2) OpenEdition

Journals

Édition électronique

URL : http://journals.openedition.org/communicationorganisation/1585

DOI : 10.4000/communicationorganisation. 1585

ISSN : 1775-3546

Éditeur

Presses universitaires de Bordeaux

Édition imprimée

Date de publication : 1 novembre 1992

ISSN : 1168-5549

Référence électronique

Valérie Carayol, « Les structures de communication », Communication et organisation [En ligne],

2 | 1992, mis en ligne le 26 mars 2012, consulté le 19 avril 2019. URL : http://journals.openedition.org/ communicationorganisation/1585; DOI : 10.4000/communicationorganisation.1585

Ce document a été généré automatiquement le 19 avril 2019

(c) Presses universitaires de Bordeaux 


\title{
Les structures de communication
}

\author{
Valérie Carayol
}

1 La relation communication/structure formelle a fait l'objet de multiples travaux en communication des organisations comme en atteste la revue de la littérature que nous proposent F. Jablin et D. Robichaud dans ce même numéro de la revue Communication \& Organisation. Les principales dimensions structurelles observées, configuration, complexité, formalisation, centralisation, et leur mise en relation avec un certain nombre de traits de la communication au sein des organisations ont donné lieu à de multiples recherches aux résultats souvent contradictoires. Si certains travaux ont mis en évidence une sorte de «déterminisme structurel» sur les modes de communication dans l'organisation, d'autres font état de résultats beaucoup plus incertains.

2 Il apparaît, au vu de ces travaux, que lorsque l'interaction structure/communication est interrogée, les politiques de communication qui peuvent avoir été mises en place par la direction de l'organisation sont très rarement prises en considération. Lorsque l'on observe, les modes de communication d'un supérieur avec ses subordonnés selon son éventail de contrôle $e^{1}$, on ne prend pas en compte l'existence éventuelle d'une politique de rémunération contractuelle basée sur des critères communicationnels, comme par exemple l'obligation de réunir son équipe au moins une fois par mois pour obtenir une prime. Ceci n'est évidemment qu'un exemple et d'autres mesures incitatives peuvent avoir été mises en place par la direction. Des priorités peuvent avoir été formulées par le haut management: utiliser les réunions plutôt que les circulaires, etc. L'entreprise et l'organisation sont souvent considérées comme des « forêts primaires », sortes de milieux naturels où il est possible d'observer la « nature » de la communication à l'état " brut ». Aussi peut-on formuler des spécificités de la communication de certaines espèces particulières, les cadres, les agents de maîtrise, les employés, lorsqu'ils se trouvent dans des milieux différents...

3 Parallèlement dans le milieu des consultants en communication, on parle de la communication comme d'une activité stratégique qui se " gère ", qui doit faire l'objet de plans stratégiques, qui doit être structurée, évaluée et pilotée. Et l'on insiste sur les 
mesures à mettre en place pour influer sur les modes de communication de certaines catégories de personnel.

Il y a évidemment discordance et paradoxe entre ces deux phénomènes. D'un côté la communication est considérée «à l'état de nature » dans l'organisation; dans l'autre, comme quelque chose d'éminemment construit, d'intentionnellement construit, susceptible de faire l'objet de politiques.

5 Les travaux qui prennent en compte l'influence des politiques de communication ou qui soulignent leur influence sur les modes de communication de l'organisation, nous viennent des chercheurs qui s'intéressent aux nouvelles technologies de communication. De plus en plus de chercheurs remettent en cause l'alternative entre un déterminisme technologique et un déterminisme social des nouvelles technologies au sein des organisations et mesurent qu'une même technique peut conduire à des pratiques et des usages fort différents suivant les politiques mises en place par la direction.

Dans un article paru dans Pour la Science ${ }^{2}$, Lee SPROULL et Sara KIESLER rendent compte d'une recherche sur les réseaux informatiques et notent que :

«Si les techniques de communication sont analogues entre les diverses entreprises,

le comportement des utilisateurs varie beaucoup selon la politique de la direction ».

Dans les réseaux, où l'accès au courrier électronique est facile et libre et où la direction considère que le coût du courrier électronique fait partie des frais généraux et n'est pas imputable aux employés ou à leur service, les employés envoient et reçoivent entre 25 et 100 messages par jour. Dans d'autres entreprises ou la direction limite l'accès au réseau ou impute les frais du courrier électronique sur le compte des utilisateurs, l'utilisation est bien plus restreinte. Les chercheurs en déduisent que :

« l'accès à un réseau n'est pas déterminé par des considérations techniques mais plutôt politiques ».

8 La prise en compte des politiques de la direction d'une organisation en matière de gestion de la communication dans la recherche nécessite toutefois une réflexion approfondie sur cette question. Or, jusque-là, les recherches sur ce point sont très peu nombreuses. Peu de travaux ont pour objet une réflexion ou une théorisation sur les pratiques de gestion de la communication dans l'organisation.

Le travail que nous allons exposer s'inscrit dans une approche de ce type. C'est une tentative pour explorer ce champ, encore peu débroussaillé. Il s'intéresse aux approches managériales de la fonction communication et en étudie un aspect, à savoir les structures mises en place pour gérer la communication dans les entreprises.

\section{Fonction communication et structures de communication dans la littérature}

10 Si la fonction communication a fait l'objet d'attention, c'est surtout à travers l'étude de ses responsables. Lors d'un récent colloque consacré à la communication des organisations ${ }^{3}$, étaient réunis autour d'une table à déjeuner quelque six Directeurs de la communication. Un rapide tour de table permit de voir qu'ils étaient Directeur marketing, Directeur du personnel. Directeur de la communication interne, Responsable de la promotion des ventes, enfin, qu'ils avaient tous des responsabilités assez distinctes, qu'ils avaient des positions fort différentes dans l'organigramme de leur organisation, et qu'ils se reconnaissaient tous sous le terme de Directeur de la communication. Cette 
grande disparité dans les statuts, les rôles, a suscité un certain nombre de travaux et d'enquêtes sur les Directeurs de communication ${ }^{4}$. Ils s'intéressent essentiellement aux hommes de communication, à leur formation, leurs profils, aux qualités requises pour le métier, éventuellement s'inquiètent de leur statut ${ }^{5}$ et de leur avenir ${ }^{6}$ assombri par la multiplication des formations à la communication et la saturation du marché de l'emploi.

11 Très peu de travaux de recherche en revanche, s'intéressent aux structures de communication et aux rapports que peuvent entretenir les structures et les stratégies de communication. L'Union des Annonceurs (U.D.A) a proposé deux enquêtes (88-92) dans lesquelles les structures de communication sont classées en quatre groupes en fonction de leurs effectifs. Quelques articles constatent une grande variété des structures de communication ${ }^{7}$, le plus souvent éclatées entre plusieurs directions de l'entreprise.

Pour certains, une structure de communication n'a pas de raison d'être, la fonction même de Directeur de la communication est inutile si chaque responsable de management fait correctement son travail. C'est le cas, si l'on en croit Olivier Postel-Vinay, d'Alain Laufenburger qui représente en France l'International Association of Business Communicators (plus de 11000 membres) qui affirme :

«Le directeur de la communication doit aujourd'hui disparaître: c'est son seul avenir et la meilleure chose à faire pour développer la communication d'entreprise ${ }^{8}$

et de Michel CROZIER ${ }^{9}$ qui dit :

«Ce qu'on appelle la communication d'entreprise aboutit souvent à un résultat nul, voire négatif. Elle se heurte, en secret, à un rejet agressif. Les entreprises sont victimes du mythe de l'expert. Le directeur de la communication est une excroissance du système bureaucratique de l'entreprise. La communication ne devrait pas faire l'objet d'une spécialisation "

Dans la presse professionnelle, il y a bien, çà et là, quelques articles qui montrent qu'il existe des querelles de territoires entre Direction du personnel ${ }^{110}$ ou Direction du marketing ${ }^{11}$ et Direction de la communication. Presque toujours, cependant, la question de la variété des structures et de la variété du positionnement dans l'entreprise des Directeurs de la communication est évacuée comme si elle portait atteinte à la légitimité d'une profession en construction, le discours majoritaire étant: cela n'a aucune importance, l'important est la cohérence de l'image. Consciente, sûrement, de cette difficulté, l'Association Entreprise et Médias, principale association professionnelle française de Directeurs de la communication a défini comme donnant droit au titre de Dircom, un certain nombre de critères : il faut être rattaché directement à la Présidence ou à la Direction générale, avoir la responsabilité des relations avec la presse et avoir au moins quatre autres responsabilités parmi les suivantes: publicité, communication interne, financière, organisation d'événements, parrainage, lobbying, études et sondages, audio-visuel...

Même si elle est très souvent évacuée du discours des professionnels de la fonction, la question des structures des services communication n'est pas pour autant dénuée d'intérêt. Les structures font partie du dispositif de gestion de la fonction et si l'on veut éclairer celui-ci, leurs études ne peut qu'y contribuer. De plus un certain nombre d'indices laissent transparaître des difficultés d'exercice des métiers de la communication dans l'organisation, liées à des phénomènes structurels. Dans une enquête effectuée par l'agence Mancom ${ }^{12}$ en 1986 auprès de responsables de communication sur les problèmes rencontrés dans la mise en place des actions de communication, $39 \%$ des personnes interrogées 
citent des problèmes de structures, en deuxième position derrière les problèmes de budget. Par ailleurs, le rattachement hiérarchique n'est pas indifférent aux responsables de communication interne, puisqu'une autre enquête ${ }^{13}$ révèle que $64 \%$ de ceux-ci souhaitent un rattachement à la Direction générale, alors que $26 \%$ sont rattachés au Directeur des ressources humaines, $56 \%$ à la Direction de la communication et $15 \%$ à la Direction générale.

\section{Les modèles explicatifs des structures de communication}

16 M.H. WESTPHALEN, à propos de la variété des configurations structurelles de la fonction communication, explique le phénomène par « la taille de l'entreprise, et le statut accordé à la communication $»^{14}$. D'autres raisons explicatives sont parfois avancées: la grande variété des structures serait due au secteur d'activité, à une stratégie de communication particulière. Ainsi dans un même secteur d'activité et à taille égale d'entreprise, on serait tenté d'adopter les mêmes structures, ou encore les structures correspondraient à des stratégies de communication spécifiques. En avançant de telles hypothèses explicatives, on se réfère implicitement au concept de rationalité tel qu'il a été développé par Max WEBER. Celui-ci repère une forme de rationalité ou "rationalité objective par justesse ", qui exprime :

« la justesse de la conduite par rapport à un type de solutions considérées comme valables dans la situation évoquée $»^{15}$.

Il existe, selon lui, un type ou un modèle idéal d'organisation qui serait le résultat de la façon dont plusieurs personnes affrontées au même type de problème finissent par trouver le même type de solution.

Deux théories des déterminants structurels développées en Sciences des Organisations sont en rapport avec cette conception de l'organisation et avec les hypothèses explicatives de la diversité des structures que nous avons évoquées.

La première, la Théorie de la contingence, développée par R. Lawrence et J.W. Lorsch ${ }^{16} \mathrm{a}$ mis en évidence l'influence de l'environnement sur la mise en place d'une structure. Ces auteurs ont montré que dans un environnement stable ou dynamique, on adoptait souvent des structures différentes. D'autres travaux comme ceux de J. Woodmard ${ }^{17}$ ont montré l'existence de relations entre technologie et structure lorsque l'on va de la production à l'unité vers la production de masse ou vers la production en continu. Ces travaux postulent que l'environnement et le contexte sont déterminants, par rapport à d'autres facteurs, comme facteurs explicatifs des variations de structures. Ils se réfèrent à une image biologique de l'organisation; elle est considérée comme un organisme qui s'adapte à son environnement.

La deuxième, la Théorie du choix stratégique, développée par I. Ansoff $»^{18}$ et A. Chandler ${ }^{19}$, a montré que, même si on ne peut pas renier un certain nombre d'effets de rétroaction entre structure et stratégie, c'est celle-ci qui est déterminante. En ce sens elle postule que la structure suit la stratégie et qu'elle est un moyen au service de celle-ci. Elle se réfère à une image mécaniste de l'organisation; celle-ci est considérée comme un mécanisme rationnel qui permet la réalisation d'objectifs formulés par les dirigeants ou la coalition dirigeante. 
21 La question des déterminants structurels a fait couler beaucoup d'encre dans les Sciences de l'Organisation et d'autres raisons explicatives ont pu être avancées qui ne sont plus basées sur ce concept de "rationalité objective par justesse»: les structures dépendraient des rapports de force et $\mathrm{du}$ jeu des acteurs de l'organisation ${ }^{20}$, des traits psychologiques des dirigeants ${ }^{21}$ elles seraient des systèmes de défense contre l'anxiété et les réceptacles des aspects psychotiques et névrotiques des individus dans les organisations $^{22}$, elles seraient des phénomènes émergents, résultats de prises de décisions chaotiques où la rationalité est hypothétique et morcelée ${ }^{23}$. Ces explications constituent une remise en cause du management considéré comme une activité rationnelle de maîtrise des actions de l'entreprise. Si elles permettent de briser l'idée d'une rationalité « objective » à l'œuvre dans le processus de gestion et détruisent l'illusion d'un modèle idéal du management, elles ne proposent pas de modèle nouveau integrable dans un projet managérial. Prendre en compte, par exemple, les névroses des individus participant à un processus de décision est certes intéressant mais délicat à mettre en œuvre. Il nous paraît donc indispensable, dans l'optique d'une meilleure compréhension des processus de gestion de la fonction communication, avant tout autre travail, d'explorer la pertinence des deux premiers paradigmes explicatifs, soit ceux de la contingence et du choix stratégique pour voir s'ils permettent de rendre compte de la réalité des structures de communication.

Dans un premier temps, nous étudierons les structures mises en place pour gérer la communication dans des entreprises évoluant dans un environnement aussi homogène que possible; dans un deuxième temps, nous essayerons de voir si les structures de communication « suivent la stratégie de communication ».

\section{Le terrain d'investigation : les Caisses Régionales de Crédit Agricole}

Le choix de notre terrain d'investigation se devait d'être adapté à l'étude des structures de communication dans un environnement homogène et nous souhaitions que les entreprises concernées aient une pratique de la communication assez ancienne et développée.

Notre choix s'est porté sur des Caisses Régionales de Crédit Agricole. Ces entreprises, qui ont en moyenne 800 salariés, sont des sociétés coopératives de droit privé, banques régionales de plein exercice, multi-services et multi-marchés. Au nombre de 85 en 1991, elles sont généralement à circonscription départementale.

Les Caisses Régionales sont actionnaires à $90 \%$ de la Caisse Nationale de Crédit Agricole, les $10 \%$ restant appartenant aux salariés du groupe. Celle-ci a par rapport aux Caisses Régionales, des fonctions financière, d'assistance, de promotion, de coordination, d'exploitation du réseau international (27 implantations dans 17 pays) et d'animation de l'ensemble des filiales spécialisées du groupe. Le groupe Crédit Agricole est actuellement la première banque française par les fonds propres, le résultat net et le bilan. C'est également la première banque européenne et la cinquième banque mondiale derrière quatre banques japonaises ${ }^{24}$.

Chaque Caisse Régionale, bénéficie, à l'inverse de certaines succursales d'autres groupes bancaires non mutualistes, d'une grande autonomie et particulièrement d'une totale autonomie d'organisation, ce qui rend notre échantillon très intéressant. En effet, ces 
entreprises constituent un groupe très homogène et parfaitement adapté à l'étude que nous comptons mener. Chacune d'entre elles est à la fois : résultante de quatre composantes, compte-tenu de la spécificité des publics liée à la forme coopérative des Caisses Régionales :

La communication marchande. Son objectif est la vente du produit ou du service et l'aide à la gestion de la relation marchande. Elle utilise comme moyen la publicité produit, le design, la communication directe, la promotion des ventes... Ses publics : le grand public, les consommateurs du produit ou service, les distributeurs, les prescripteurs...

La promotion institutionnelle. Son objectif : accroître la notoriété, souligner son rôle social et économique, mettre en avant la mission et les valeurs. Elle utilise comme moyens, la communication de prestige, d'opinion, de recrutement, financière, le parrainage, mais également les moyens relationnels, les relations presse et relations publiques. Ses publics: le grand public, et des publics spécifiques dispersés, les actionnaires, les financiers, les publics de proximité, les leaders d'opinion, les groupements divers, les corps constitués, la presse...

34 - La communication salariée. Son objectif : gérer les communications avec les salariés et, selon les cas, impliquer, expliquer, relier, informer, concerter, faire participer etc.. Elle utilise comme moyens des outils d'écoute, le pilotage de projets, le travail en groupe, les outils d'informations, les clubs professionnels... Ses publics : les salariés, les responsables de filiales etc..

35 - La communication élus. Son objectif : gérer les communications avec les membres élus de la structure mutualiste, les administrateurs de Caisses Locales. Cette autre forme de " communication interne" peut avoir des objectifs et des outils proches de ceux de la communication salariés. Nous avons retenu comme éléments de description d'une structure de communication, deux traits structurels qui nous ont apparu adéquats à la description d'une fonction 
particulière de l'entreprise. La plupart des traits structurels qui servent à la description des structures ont été bâtis, en effet, pour décrire des structures d'entreprise et non des structures de fonction.

37 - La départementalisation., ou choix de regroupement des activités. L'étudier revient à se poser la question: les unités concourant à la mise en œuvre des composantes de la communication sont-elles regroupées ou non et quel est leur agencement?

- Le rattachement hiérarchique. L'étudier revient à se poser la question : à quelles entités les unités concourant à la mise en œuvre des composantes de la communication sont-elles rattachées ? Direction générale, du personnel, marketing, etc. ?

L'investigation a été conduite à partir de l'analyse de documents, en l'occurrence l'organigramme général de l'entreprise et l'organigramme plus détaillé des unités concourant à la mise en œuvre de la fonction communication. Cette méthode nous semble introduire moins de biais que la méthode utilisant des questionnaires auto-administrés, d'autant que, lorsqu'il y a partage des responsabilités de communication, le problème de savoir qui répond au questionnaire devient très important. Chaque Caisse Régionale du Groupe Crédit Agricole a été sollicitée pour l'envoi de ces documents et s'est montrée très souvent extrêmement coopérative. Le taux de réponse a été très bon puisque nous avons reçu 54 réponses (soit $60 \%$ des entreprises contactées) parmi lesquelles 47 étaient totalement exploitables. Nous avons pu, par ailleurs, vérifier que les entreprises qui participaient à l'étude constituaient réellement un groupe homogène en comparant des données de structure (nombre de salariés, nombre de bureaux, nombre de Caisses Locales) et des données d'environnement (taux de concurrence bancaire) dont on a calculé la moyenne et l'écart-type.

40 Le choix de l'organigramme comme outil de référence s'explique. Si celui-ci ne reflète pas l'organisation réelle de l'organisation, comme de nombreux travaux l'ont montré, il n'en constitue pas moins une image intéressante. Au niveau de la forme structurelle, il donne une image assez exacte de la division du travail et du regroupement des postes. C'est comme l'indique $\mathrm{H}$. Mintzberg ${ }^{26}$, une sorte de carte géographique précieuse pour repérer les villes et les liaisons routières mais donnant peu d'indications sur le potentiel économique et régional. L'organigramme est le miroir du système formel tel qu'il a été décidé officiellement.

\section{La départementalisation}

Les composantes de la communication que nous avons distinguées sont, à une exception près - une seule entreprise de l'échantillon n'a pas de service de communication salariés - toujours décelables dans l'organigramme de l'entreprise.

Si on observe les modes d'agencement des différentes composantes de la communication entre elles, on ne trouve pas moins de 10 configurations mises en œuvre. Trois configurations correspondent néanmoins à la situation de 35 entreprises sur 47 soit les trois quarts de l'échantillon.

Ce sont les configurations suivantes :

1 - Toutes les composantes sont réunies (16 entreprises)

2 - Tout est réuni sauf la communication marchande (11 entreprises) 
3 - Les communications internes - salariés et élus - sont réunies ainsi que communication marchande et la promotion institutionnelle, mais dans deux unités différentes (8 entreprises).

La première constatation à faire est que toutes les entreprises de l'échantillon ont mis en place une unité distincte pour gérer la communication avec un public particulier au secteur coopératif: les administrateurs élus. La communication élus n'est jamais assimilée à la communication salariée, seule souvent à recevoir le qualificatif de communication interne. La mise en place d'une unité particulière correspond apparemment à un besoin, si ce n'est une nécessité et sur ce point la forme coopérative des Caisses Régionales est en toute évidence déterminante. Sur la structuration proprement dite et la départementalisation en particulier, la diversité des solutions adoptées par chacune de ces entreprises ne plaide pas pour une influence déterminante du contexte et de l'environnement.

\section{Le rattachement hiérarchique des différentes composantes de la communication}

Pour chaque composante de la communication, nous indiquons figure 1 le nombre de cas où le rattachement a été observé.

\begin{tabular}{|l|c|c|c|c|}
\hline Rattachement & $\begin{array}{c}\text { COM } \\
\text { Marchande }\end{array}$ & $\begin{array}{c}\text { PROMOTION } \\
\text { Instit }\end{array}$ & $\begin{array}{c}\text { COM } \\
\text { Salariés }\end{array}$ & $\begin{array}{c}\text { COM } \\
\text { Sociétaires }\end{array}$ \\
\hline $\begin{array}{l}\text { DG/DGA } \\
\text { Secrétarotat } \\
\text { Général }\end{array}$ & 8 & 19 & 19 & 20 \\
\hline $\begin{array}{l}\text { Direction } \\
\text { comprenant } \\
\text { les R.H. }\end{array}$ & 3 & 7 & 15 & 13 \\
\hline $\begin{array}{l}\text { Direction } \\
\text { Marketing ou } \\
\text { de marché }\end{array}$ & 33 & 15 & 8 & 9 \\
\hline $\begin{array}{l}\text { Autre } \\
\text { Direction }\end{array}$ & 3 & 6 & 4 & 47 \\
\hline Total & 47 & 47 & 46 & 5 \\
\hline
\end{tabular}

Fig 1. Rattachement hiérarchique des composantes de la communication

Ces résultats montrent qu'il existe également une grande variété des rattachements des différentes composantes même si de grandes tendances sont décelables:

- la communication marchande est rattachée dans $70 \%$ des cas à une Direction comprenant le marketing ou une direction de marché, 
51 - la promotion institutionnelle le plus souvent rattachée aux instances de direction (D.G/ D.G.A/Secrétariat général) soit dans $40 \%$ des cas, mais aussi également à une Direction comprenant le marketing ou une Direction de marché, dans $32 \%$ des cas,

52 - la communication salariés est rattachée aux instances de direction dans $41 \%$ des cas et à une Direction comprenant les ressources humaines dans $32 \%$ des cas,

53 - la communication élus connaît à peu près le même rattachement puisqu'elle est rattachée aux instances de Direction dans $42 \%$ des cas et à une Direction comprenant les ressources humaines dans $27 \%$ des cas.

54 La logique veut que deux entreprises ne peuvent avoir le même rattachement de toutes les composantes de la communication que si elles ont également la même départementalisation. Si l'on associe les deux paramètres, départementalisation et rattachement, les entreprises qui ont fait à peu près le même choix se comptent sur les doigts de la main.

55 Si l'on observe ces résultats, force est de constater que dans un environnement similaire, les différentes solutions de structuration adoptées ne répondent pas à un modèle unique. L'hypothèse explicative de la variété des structures construite à partir de la Théorie de la contingence ne semble donc pas apte à rendre compte de la réalité étudiée.

\section{Les structures sont-elles le fruit d'une stratégie de communication?}

Cinq entretiens avec des directeurs généraux de Caisses Régionales de Crédit Agricole, ayant opté pour la même départementalisation de leur fonction communication - toutes les composantes de la communication sont regroupées - nous ont permis de mettre à jour quelques-unes des raisons à l'origine de la mise en place de la structure et d'essayer de voir si elles relevaient d'une stratégie de communication.

57 Au cours de ces entretiens de type analyse biographique/analyse rétrospective de l'action, les directeurs devaient retracer l'histoire du ou des services communication, les modifications qu'ils avaient apportées à leur arrivée dans l'entreprise à la fonction communication, etc..

La définition opératoire de la stratégie utilisée est celle de R. A. Thietart T :

«La stratégie est l'ensemble des décisions et des actions relatives au choix des moyens et à l'articulation des ressources en vue d'atteindre un objectif $»^{27}$

Les raisons évoquées par les directeurs généraux sont considérées comme relevant d'une stratégie de communication, si elles sont mises en rapport avec un objectif assignable à la communication.

60 Tout d'abord, notons que pour deux dirigeants, les structures de communication ont peu d'importance, la qualité de la communication d'une entreprise étant, selon eux, bien plus imputable au comportement de l'encadrement et de l'équipe de direction, qu'aux actions d'un service communication. L'un d'entre eux a d'ailleurs opté pour la structure de son prédécesseur, sans y apporter de modification.

61 Pour deux autres directeurs généraux, au contraire, les structures sont extrêmement importantes. Pour l'un, seules une organisation et une structure bien pensées permettent une action cohérente, surtout en matière de communication, pour l'autre, elle est importante au point de faire des changements de structure un principe de management. 
Il faut selon lui changer fréquemment de structure pour maintenir le dynamisme des équipes. Une structure qui a vécu quatre ans a, selon lui, déjà perdu sa capacité dynamisante.

62 Ces commentaires sur les structures mis à part, il apparaît que les raisons qui sont intervenues dans le choix de la structure sont diverses.

63 Des raisons financières sont évoquées par deux dirigeants; le regroupement des composantes de la communication permettrait des économies, dans un secteur « où l'on peut toujours rajouter du monde ». Le choix est alors surtout un choix budgétaire.

Des raisons liées à l'optimisation des Ressources Humaines sont également citées. Il faut des hommes disponibles pour « incarner » la structure. L'organisation doit optimiser les Ressources Humaines disponibles et la mise en place d'une structure doit prendre en compte ce phénomène.

65 Des raisons liées à un choix d'implication personnelle sont aussi mentionnées. Le souhait de s'impliquer personnellement implique parfois d'avoir le service communication en ligne directe.

66 Des raisons liées à la communication et que nous considérons comme relevant d'une stratégie de communication sont également citées. Elles sont de plusieurs natures et ont été évoquées par quatre directeurs généraux, même si elles n'étaient pas en première position. Ce sont :

67 - l'importance symbolique : créer un service communication important, cela aide à montrer le souhait de dialogue de la direction,

68 - la cohérence des messages : tout regrouper permet de " parler d'une même voix ", et donc d'avoir une communication plus cohérente,

69 - l'aspect facilitateur (ou entravant) de certaines positions dans l'entreprise pour assurer une mission de communication. Ainsi le choix du rattachement hiérarchique de la fonction aux Ressources Humaines ou à la Direction générale poserait des problèmes particuliers alors que d'autres rattachements faciliteraient les missions.

70 La première constatation à faire est que les cinq chefs d'entreprise ont mis en place la même départementalisation de la fonction communication pour des raisons sensiblement différentes. Le choix de la structure de communication paraît, par ailleurs, obéir à des logiques variées, qui concernent le niveau global de l'entreprise, et pas seulement à une logique liée à la stratégie de communication. Si pour un directeur sur cinq, le choix de la structure de communication n'est pas en liaison avec une stratégie de communication, puisqu'il n'a pas été mis en rapport avec un objectif lié à la communication, pour les autres, elle est intervenue comme au moins un des facteurs à prendre en compte.

71 En ce sens, il est certain qu'on ne peut attribuer à la stratégie de communication le qualificatif de facteur déterminant pour l'adoption d'une structure mais, tout au plus, celui d'un des facteurs l'ayant influencée.

\section{Vers l'étude des rétroactions de la structure sur la stratégie de communication.}

Si la stratégie de communication n'est pas un facteur déterminant dans l'adoption d'une structure de communication, on ne peut cependant pas conclure qu'il n'existe aucun lien entre stratégie et structure, à l'issue de l'analyse de ces entretiens. Si le contexte et 
l'environnement ne sont pas déterminants dans les choix de départementalisation de la fonction ou dans les choix de rattachement hiérarchique des composantes de la communication, on ne peut cependant pas conclure à l'indépendance totale entre environnement et structure puisque toutes les entreprises de l'échantillon ont mis en place une unité spécifique pour gérer la communication avec les administrateurs élus. Il y a là indéniablement une spécificité du monde coopératif. En définitive, ces deux études montrent qu'il existe une multiplicité de facteurs intervenant dans l'adoption d'une structure de communication, sans qu'aucun n'apparaisse comme réellement déterminant.

Progresser dans l'analyse implique, en toute évidence, de renouveler la problématique envisagée et de sortir d'un cadre de causalité déterministe. Un point évoqué au cours de ces entretiens nous semble particulièrement intéressant, c'est celui qui concerne l'aspect facilitateur ou entravant de certaines positions dans l'entreprise pour mener à bien des missions de communication. Il remet en avant le problème des interactions stratégies/ structures sous un jour nouveau et permet de relancer l'interrogation sur les structures sous un angle dynamique, en envisageant la structure comme un processus et un cadre à la fois « habilitant et contraignant ${ }^{28}$. Dans cette optique un intérêt plus soutenu devra être porté aux interactions et rétroactions de la structure de communication sur les stratégies de communication.

\section{NOTES}

1. Nombre de subordonnés.

2. Spoull L., Kiesler S., «Réseaux Informatiques et organisation du travail » in Pour la Science, $\mathrm{n}^{\circ}$ 169 , novembre 1991, pp. 82-90

3. Colloque "Éthique et communication », novembre 1990, Institut de Science de l'Information et de la Communication, Bordeaux 3

4. Guerin, S.« Directeurs de la communication, état des lieux in Médias Pouvoirs $\mathrm{n}^{\circ} 18$, avril/mai/ juin 1990, pp. 73-80.

5. Tixier M., «Évolution de la fonction communication, ou comment la sortie d'une prétendue impasse. Quelque conseils à la $3^{\mathrm{e}}$ génération de Dircoms » In Humanisme et entrepris $\mathrm{n}^{\circ} 191$, février 1992, pp. 73-90».

6. Verge J.-P. «Bernard Krieff : peu de patron sont satisfait de leur DIR-COM » In Stratégies $\mathrm{n}$ - 755, 9/09/91.

7. FNEP, Fondation Nationale entreprise et performance, «La communication interne et externe dea grandes entreprises" In Pangloss $n^{\circ} 19,1989,244$ in Dossier «Entreprise et Communications », Hors-série, septembre 1991, Les Échos

8. Cité par Postel-Vinay O. « La communication au banc d'essai » In Dynasteura, fev. 1990 pp. 59-63

9. Idem

10. JordanJ.,-L'information et la communication sont-elles du ressort de la fonction personnel? in Ressources Humaines ${ }^{\circ}$ 6, janvier 1987, pp 7-19

11. Verges, JP.-BKC sonde les Dircoms » in Stratégies, $\mathrm{n}^{\circ} 775,10 / 02 / 92$

12. Citée dan Communication et Business $n^{\circ} 4$, oct. 1986, p. 45 
13. Citée dans L'usine Nouvelle, $\mathrm{n}^{\circ} 2273$, du $21 / 06 / 90$

14. Westphalen, M.H., Le communicator, Guide opérationnel pour la communication d'entreprise, Paris, Bordas (Dunod), 1989

15. Weber, M.. Essais sur la Théorie de la Science, Paris, Pion, 1965, cité par Salnsaulieu R, Sociologie de l'organisation et de rentre-prise, Paris, FNEP, Dalloz, 1987.

16. Lawrence R, Lorsch J. W, Adapter les structures de l'entreprise, intégration ou différentiation Paris Éd. d'Organisation, 1973.

17. Woodmard J., Industrial Organization, théorie and practice, London, Oxford University Press, 1965

18. Ansoff, I.Stratégie du développement de l'entreprise, Paris, Éd. Hommes et Techniques, 1968.

19. Chandler, AD, Stratégies et structures de l'entreprise, Paris, Éd. d'Organisation, 1972.

20. Crazier M., Friedberg E. L'acteur et le système, Paris Seuil, 1977.

21. Miller D., Dröge C., "Psychological and traditionnel determinants of structure, in Administrative Science Quaterly, 31/4,1986, pp. 539-560.

22. Conception d'Ellott Jacques du Tavistock Institute exposée par Enriquez Eugène, « Management et psychanalyse » in Sciences Humaines, $\mathrm{n}^{\circ} 5$ avril 1991 pp. 22-23

23. Cohen, M.D., March, J.G., Olsen J.-P. «A garbage can model oí organizational cholee In Administrative Science Quaterly, vol 17,1972, pp. 1-25.

24. «Les quinze première banques mondiale » in AGEFI du 19/08/92.

25. Flipo, J.-P.. Le management des entreprises de service, Paris, Éd. d'organisation. 1984, 252 p. bibliogr.

26. Cf. Clauzel, B. «Les structures de l'entreprise " in Les Cahiers Français n² 233, déc. 1987, pp. 19-25

27. Thiétart R.A., La stratégie d'entreprise, 1 Éd. Perta, Mac Graw Hill, 1984, p 1

28. Eraly A., La structuration de l'entreprise: la rationalité en action, Bruxelles, Éditions de l'Université, 1988

\section{RÉSUMÉS}

Cet article fait état d'une recherche menée dans le cadre d'une réflexion sur les modes de gestion de la fonction communication. Il présente les résultats d'une enquête menée dans 47 entreprises bancaires du secteur coopératif, des Caisses Régionales de Crédit Agricole. L'étude cherche à répondre à deux questions. L'environnement a-t-il un effet structurant sur l'organisation de la fonction communication? Les structures de communication dépendent-elles d'une stratégie de communication?

\section{AUTEUR}

\section{VALÉRIE CARAYOL}

Valérie CARAYOL est allocataire de recherche du Ministère de la Recherche et de la Technologie et membre du Groupe de Recherche en Communication des Organisations de l'Université de Bordeaux 3 où elle enseigne. Elle travaille sur les modes de gestion de la communication et la modélisation des stratégies de communication interne. 